\title{
Cosmology and Gravitation
}

\author{
M. D. Maia \\ Universidade de Brasilia, Instituto de Física, 70919-970, Brasilia DF, Brazil
}

Received on 27 November, 2003

This is a brief summary with comments on selected contributions to the Cosmology and Gravitation section at the $24^{\text {th }}$ Brazilian Meeting on Particle and Fields (ENFPC XXIV), held at Caxambu, from September 30 to October 4, 2003.

\section{History and Trends}

The earliest known publication on Einstein's Relativity in Brazil is the little book by Amoroso Costa back in 1922 [1]. After that, a more technical book by Pedro Rache appeared only in 1932 [2]. Research papers properly may be tracked back to the early forties, with the collaboration between Mario Schönberg and Chandrashekar in Chicago [3]. Regular and systematic academic programs similar to the ones we have today, leading to graduate degrees, started in the mid sixties, with F. Kremmer in the state of Paraná and with C. Oliveira at the Centro Brasileiro de Pesquisas Fisicas (CBPF) in Rio de Janeiro. This gives us an estimate of approximately 40 years of development of an effective academic research on cosmology and gravitation (CGR) in Brazil.

During this period we have grown to approximately 150 researchers in that area, scattered through almost all states of Brazil. The present meeting counted with a total of 84 contributions in CGR alone, including two invited plenary talks, three parallel presentations, 20 short oral communications and 59 poster presentations. Here we present a sampled analysis of these contributions, assembled in just a few categories.

There is a noticeable tendency towards cosmology, with 53 contributions, against a only 31 from topics in local gravitation, a clear demonstration of a new trend. This is hardly surprising, considering the present status of cosmology as a highly experimental and interdisciplinary part of physics, which has provided us with a vast and challenging collection of data waiting for explanations.

Therefore, it is also no surprise that the two invited plenary talks on CGR were on the main experimental results of observational cosmology, the accelerated expansion and the cosmic background radiation (CMBR).

The first plenary talk by A. Lassenby (Cavendish): Cosmic Microwave Background - Recent Results and Their Implications for Cosmology, analyzed the results on the CMBR from the Wilkinson Microwave Anisotropy Probe (WMAP) experiment released early this year.

After reviewing the present observational situation, it was pointed out that those results confirm previous measurements of the COBE satellite and more recent balloon and ground-based experiments, but evidenced a deficit of fluctuation power on the largest scales, and also a variation of the spectral index in the primordial perturbation. In his own conclusion, "these measurements have confirmed the parameters of the current 'concordance model' of the Universe, but with some intriguing pointers towards "new physics'" [4].

The second plenary talk by Jerome Martin (Inst. d'Astrophysique de Paris) Inflation and Precision Cosmology, reviewed the inflationary mechanism in cosmology and its consequences to the CMBR anisotropy and formation of large scale structures. The predictions of inflationary theoretical models were compared with the WMAP data, showing an agreement between them. The talk also included an interesting discussion on the trans-Planckian problem of inflation. That is, the fact that all the scales of astrophysical relevance today were, at the beginning of inflation, smaller than the Planck length. Finally, possible observational signatures, in particular in the CMBR anisotropies, leading to topological definitions were also discussed [5].

These two talks have set the background for the great majority of the discussions on Cosmology during the meeting. Therefore, it is natural to start with cosmology, moving towards the local aspects of gravitation.

\subsection{Essences of Inflation}

A number of possible answers to two of the major open questions in today's cosmology were presented: what caused the past inflation and what causes the current accelerated expansion of the universe?

The presently popular quintessence model features a slowly decaying scalar field associated with a phenomenological potential with a small energy density, of the order of today's value of the Hubble parameter $H_{0} \sim 10^{-42} \mathrm{GeV}$ [6]. One known criticism to this model is that it is difficult to conciliate such small repulsive new force with the present efforts to solve the hierarchy problem for fundamental interactions [7]. Therefore, it is natural to ask if it is possible to build a quintessence model starting from the phenomenology, for example using the known data on the dynamics and thermal history of the universe, instead of postulating a potential, [8]. 
Along a similar line, we could also look for a possible relation between the primordial inflationary period and today's accelerated expansion. Using the quintessence model, it has been conjectured that both accelerated periods are described by the same scalar field, with a particle production associated with a non-zero coupling between matter and the inflaton [9].

On the other hand, particle creation associated with inflation can be explained in different ways. For example, it may be considered as a result of the gravitational field through an irreversible process, or at the expense of the scalar field together with a phenomenological friction term [10].

It is also possible to suppose that that inflation started at finite temperature, leading to a super cooling during the first inflation phase, remaining cool till reaching the end of that phase, followed by a reheating period [11].

The non-linearity of the decay of inflation as a cause of the reheating of the universe was described as a long time regime dynamical system, characterizing a turbulent state with a non-linear transfer of energy and the consequent thermalization of the created matter [12].

The Chaplygin gas model proposes a unified description of dark matter and dark energy with a single substance specified by a hyperbolic state equation $p=-C / \rho$, [13]. The observational constraints on such fluids using data from the supernova type I (SNIa) experiments, the statistics of gravitational lensing and the FR $2 \mathrm{~b}$ radio galaxies were also studied, showing the best fits for the situations close to the model composed by the cosmological constant plus cold dark matter $(\Lambda C D M)$ limit [14].

\subsection{Topology and Topological Defects}

The experimental detection of the topology of the universe and the compatibility of topological defects with experimental data was discussed in several occasions during the meeting. In particular the question concerning the determination of the topology of the universe in a theory that is based exclusively on its local Riemannian metric geometry, as it is the case of General Relativity, leads naturally to the question on how to interpret any possible experimental measurements on the topology of the universe [15]. The finiteness of the universe has been academically discussed for a long time. Back in 50BC, Lucretius [16] wrote:

\section{“...the universe is not bounded in any direction. If it were, it would necessarily have a limit somewhere. But clearly a thing cannot have a limit unless there is something outside to limit it..."}

However, in face of the recent observational data from the WMAP experiment, it has been estimated that the universe may be finite or composed of finite cells. In fact using that data, it was recently announced that the topology of the observable universe can be that of a Poincaré dodecahedron [17]. The confirmation of such hypothesis still depends on the detection of pairs of intersection circles which should necessarily appear in such topology. This is expected to be confirmed in the next planned experiments on the CMBR.
On the other hand, the same observations suggest that the universe is nearly (spatially) flat, with the ratio $\Omega=$ $\rho / \rho_{c r}$ of the total energy density $\rho$ to the critical energy density $\rho_{c r}$ being very close to one. This is compatible with the predictions of standard inflationary models which also predicts this ratio to be closer to one by several orders of magnitude. Such analysis has been suggested as a way to distinguish between two cosmic topologies as opposed to the usual detection based on pattern repetition. This analysis may decide which topologies are potentially detectable but not excluded by observation [18].

Since The Chapligyn gas behaves sometimes as attractive and sometimes as repulsive depending on the value of the parameter $C$, it has been conjectured that it must be also associated with a topological change in the universe. At least one analysis indicates that the delectability of the topology of a universe dominated by such gas is very unlikely [19].

Previous measurements on the CMBR have indicated some incompatibility with the cosmic strings structure. This was confirmed by the WMAP experiment as mentioned in the plenary talk by Lassenby. Nonetheless, some sophisticated efforts have been made to place cosmic strings back on the track:

In one example the cosmic string structure may be modified by the addition of an electrical current, such that it would adjust to the measurements of the CMBR. This can be implemented within the scalar-tensor context, showing that the inclusion of electromagnetic properties induces logarithmic divergences [20].

In addition, torsion may also be included in a currentcarrying cosmic string. The joint effect of current and torsion would change the gravitational force and the geodesic equation of a test-particle moving around such "screwed" string [21].

In a different approach to the same problem, the cosmic string was altered by the inclusion of a dislocation. However, in doing so, an observer traveling along such string with increasing velocity, would eventually witness the formation of a time machine. This apparently undesirable effect can be fixed by considering a quantized scalar field around the string [22].

\subsection{Quantum Cosmology}

A time independent quantum gravity program based on the Wheeler-DeWitt (WDW) equation is generally known as Quantum cosmology (QC) [23]. Since in this case time does not exist, in one interpretation the solutions of the WDW equation can be seen as being timeless (or eternal so to speak). Nonetheless, to make sense with the classical limit of the theory, an appropriate classical time variable must be recovered somehow. In some models, the evolution of a classical field attached to the system sometimes is used as a time marker. However, generally speaking the recovery of time in QC remains an open problem [24].

Quantum Cosmology describes quantum states of the universe and the probability transition between these states. Therefore, it may also associate topological changes 
in the corresponding classical universe. We could speak of a quantum topological transition in "quantum topology" associated with the quantum tunneling. Some estimates show that such topological transition between homogeneous and isotropic hypersurfaces with different Riemann curvatures can be characterized by the Greens' function of the system [25].

With the eventual recovery of the notion of classical time and with the notion of topological transition, we may ask what QC would say about the recent measurements of the CMBR and of the accelerated expansion of the universe [26]. One possible answer is through the imposition of a classical variational principle. Using the Schultz variational formalism, the notion of time can be introduced so as to obtain the FRW model by a superposition of discrete stationary solutions to the WDW equation. Numerical simulations appear to agree with results obtained through timeindependent perturbation theory. It turns out that these models do not have singularities but they exhibit quantum tunnelings. Since this may imply in the loss of unitarity, the probability transition between states of the universe is not necessarily conserved, suggesting that the "many-worlds" interpretation could be applied to QC [27].

\subsection{Gravitation in Higher and Lower- Dimensions}

The four-dimensionality of space-time is an experimental fact. At least that is what we conclude from Mikowski's own words [28]

"The views of space and time which I wish to lay before you have sprung from the soil of experimental physics and therein lies their strength. They are radical... "

Of course, he was referring to the Michelson-Morley experiment and at that time nothing else would indicate that we need more than four dimensions. However, theory, experimental high energy physics and astrophysics have evolved enormously since that period, suggesting that we may need more than four dimensions to describe space-time.

In fact, gauge field theory is consistent with the addition of a set of parameters associated with a symmetry group which exists together with the Poincarè symmetry. This characterizes an "internal space" which is observable through high energy physics experiments. Therefore, today we could rightfully repeat Minkowski's words, stating that "higher dimensional physics also sprigs from the soil of experimental physics". The difficulty lies in finding the correct way to combine all degrees of freedom in a single space with dimension greater than four.

One of the most recent proposal in that direction is the scheme of "large extra dimensions", also known as the brane-world program, where the four-dimensional spacetime is a submanifold embedded in a higher dimensional space $[29,30]$. The extra dimensions can be as large as a micrometer and there are serious experiments under way to verify Newton's gravity at short distances [31]. Additional experiments are being planned for 2007, aiming to probe the extra dimensions at the $\mathrm{TeV}$ scale of energies [32].

The accelerated expansion of the universe as an obser- ved fact has been also understood as an experimental evidence for the existence of large extra dimensions. This interpretation is possible because large extra dimensions suggest a modification of Friedmann's equations by an additional term. In most models this term can is proportional to the square of the energy density [33], which does not agree with the formation of light elements from the big-bang nucleosynthesis, suggesting a number of rather creative fixes.

One of these repairs consists in modifying the energy scale of the nucleosynthesis. Admitting that the fundamental scale is $1 \mathrm{TeV}$ and using a semi-analytic procedure, the primordial Helium abundance appears to be in agreement with the observations [34].

A higher dimensional Minkowski space has also been considered as the host space (the bulk) in which the braneworld moves as an extended object [35]. In this case, the bulk, may become a source of gravitational waves itself. In the case of bulk waves produced by the linear perturbations of the bulk, we obtain besides spin two fields, other gravity related objects whose nature depends on the number of extra dimensions. Therefore, the higher-dimensional gravitational dynamics may produce a difference relative to the usual four-dimensional one when counting the spin degrees of freedom [36].

The dependence of the dynamics of an object on the dimension of the space-time also appears in different contexts. For example, it has been shown that the emission rate for a source with constant proper acceleration in a Ddimensional Minkowski space-time, minimally coupled to a massless scalar field, is equal to the emission plus absorption rates when the static source is in a thermal state. The response rate is generally proportional to a certain power of the sources constant proper acceleration, which depends on the dimension $D$ of the space-time [37].

The Aharonov-Bohm effect indicates that the electromagnetic potential is an observable. By extension a gauge potential is also taken to be an observable[38]. Therefore, looking way ahead at a quantum version of brane-worlds, as realized through the Ashtekar-Smolin loop quantum gravity program [39], we may ask if the potentials derived from the holonomy of a triad in a brane would also be an observable in the sense of the gravitational analogue to the AharonovBohm effect. An estimate of such effect for the cases the Schwarzschild and AdS-Schwarzschild space-times, in the five-dimensional Randall-Sundrum scenario, was presented [40].

Since the brane-world program sits at the interface between gravitation and field theory, some of the contributions on brane-words and applications to cosmology also appeared in the field theory sections of the meeting, where questions relative to the presence of massive Kaluza-Klein modes were discussed. In one talk massive gravitons are considered in thick branes regarded as domain walls associated with tachyon potentials. In the application to cosmology, these potentials can be selected so as to describe some form of dark energy. In particular a tachyon matter with negative pressure is suggested as a candidate for quintessence [41]. In another talk, the experimental constraints to the brane-world program, associated with the linear gravita- 
tional waves emitted by the bulk and the presence of massive Kaluza-Klein graviton modes were discussed [42, 43].

Quite on the opposite direction, there were a number of presentations on lower dimensional gravity. For example, a perfect fluid with a kinematic self-similarity has been considered in $2+1$ dimensions with circular symmetry. In this case, some solutions of Einstein's equations represent gravitational collapse in which the final state can be either a black hole or a null singularity [44].

A study on inflationary cosmology in $2+1$ dimensions, including dark energy solutions, show that matter and or radiation would interact with the inflaton field through particle production during the inflationary regime. In the present era, the interaction of matter and a dark energy component represented by the scalar field produce a positive acceleration as a function of the total pressure of the constituents [45].

A similar idea can also be applied to the case of $1+1 \mathrm{di}-$ mensions, with a scalar field representing the dark energy, where a comparative analysis is made for both the JackiwTeitelboim and the dilatonic models in arbitrary gauges [46].

\subsection{Gravitational Wave Detection}

The experimental program of gravitational wave detection in Brazil started in the early nineties with the "Graviton Project". Later on in 2000 it led to the Schenberg project for the construction of smaller spherical antennas, which consists of the current experiment, mostly developed at the National Institute for Space Research (INPE).

The spherical detectors (there are three of them) with $60 \mathrm{~cm}$ diameter have a resonance frequency of $3.8 \mathrm{~Hz}$ which is greater than the laser interferometry antennas. The 1.15 Ton copper-aluminum sphere and its 0.5 Ton vibration isolation system has been cooled down to $2 \mathrm{~K}$ for a first cryogenic test.

An initial test run has been already made and the first operational run at 4-5 K, with resonant frequency at around $3.2 \mathrm{kHz}$, is expected to operate by mid 2004, with nine transducers [47].

A simulation of the detector was made with the wave generated by two inspiralling neutron stars which eventually collide into a black hole. The signal-to-noise ratio was calculated as a function of frequency for the simulated signals, showing that the sphere is sensitive enough to detect that kind of signal up to a distance of $18 \mathrm{kpc}$ [48].

The development and construction of the transducers represents an additional engineering task, which has benefited from the experience from previously existing antennas. The present development uses a modification of the transducer used for the gravitational wave bar antenna Niobe, making it easier to achieve the standard quantum limit of sensitivity [49].

\subsection{Analog Models}

The idea behind analog models consists in getting insights on problems defined in a given branch of physics by comparison with an analog problem in another area of physics or, more generally, of science [50]. In the particular case of gravitation, one well known example is given by the thermodynamics of black holes, where Hawking's radiation is regarded as the analogous to a thermal radiation [51].

In another analogy with optics, Fresnel's equation can be written in a covariant form aiming to an analogy with wave propagation in curved space-times. The solutions of that covariant equation are intrinsically obtained, showing that the light rays propagate in eigenmodes with linear polarization [52].

An interesting example is the analogy of dark energy in cosmology with a classical fluid with negative pressure. It is noted that while in non-relativistic physics negative pressure is associated to cohesive forces, in relativity it may prevent the collapse of a mass distribution [53].

We expect that these analogies work in both ways, specially on fundamental issues. For example, we may regard the geometric description of Yang-Mills theory as analogous to Einstein's gravity. Although the latter is not a gauge theory, it seems possible to derive an equivalent gauge theory (see below). The same equivalent gauge theory may offer an interesting analogy with solid state physics, perhaps with some interesting geometrical tools.

\subsection{Alternative Formulations}

In the past years there has been a noticeable interest the development of the so called Teleparallel Equivalent of $\mathrm{Ge}$ neral Relativity. Basically, instead of using curvature this formulation of Einstein's gravitational theory uses only the torsion. The Riemann curvature of the space-time vanishes and everything else, including geodesic deviations are handled in terms of the torsion tensor. The complete equivalence between teleparallel gravity and Einstein's gravity depends on the equivalence principle but that seems to be undecided [54]. If the total equivalence holds, then this new formulation may not offer much at the classical level, including possible explanations for the dark matter and energy problem.

In the past, equivalent formulations of Einstein's theory like the two-component spinor formulation or the Newmann-Penrose formalism, have offered some simplifications on particular issues such as the Petrov classification and on the computer-algebra methods of solving Einstein's equations. Later on the spinor formalism and conformal invariance led to the development of the conformal spinor or "twistor" program, which eventually led to the concept of spin networks.

It is possible that the contribution of the teleparallel program rests not on the classical physics but on quantum gravity. Indeed, a major conceptual problem when we want to place all fundamental interactions in the same basket is that Einstein's gravitation does not behave as a gauge theory. However, as it has been reported in the meeting, teleparallel gravity appears to be a true gauge theory of gravity of the Poincarè translational group $T_{4}$ [55]. In this case we could in principle quantize gravity through the $T_{4}$ connection, bearing in mind that the $T_{4}$ group is not an easy one. Besides being non-compact, it has the rather undesirable property of 
acting nilpotently over the other symmetries, possibly leading to an acute No-Go problem when the $T_{4}$ group is in an unbroken combination with the standard gauge groups $[56,57,58]$.

\subsection{Singularities and Black Holes}

Most of the contributions on exact solutions of Einstein's equations concern the addition of a scalar field and to explore the its quantum and classical properties, notably in black-hole physics. For example, Hawking's radiation can be determined by means of a massless scalar field. In the particular cases of Schwarzschild and Schwarzschild-de Sitter space-times, the response rates at the tree level in terms of an infinite sum of zero-energy field modes can be calculated in a closed form in de Sitter space-time, but not in the in the Schwarzschild-de Sitter case [59].

The study of properties of black hole physics like the decay of its mass and the area spectra cannot be complete without the analysis of quantum gravity. Although such theory does not exist as yet, an estimate of some of these properties can be obtained from the basic properties of a given quantum gravity scheme.

For example, loop Quantum Gravity (LQG) is based on the auxiliary variables program originally developed by A. Ashtekar. A connection associated with the holonomy group of closed loops may be quantized as a $S U(2)$ gauge theory, inducing the quantum fluctuations of the metric geometry, while keeping intact the diffeomorphism invariance of the four dimensional theory [39]. Even if one such theory is far from complete, it has been claimed to contribute to the spectra of black hole area [60].

In an analogous problem but using a more traditional approach to quantum gravity, the Arnowitt-Deser-Misner (ADM) quantization, together with the de Broglie-Bohm interpretation of quantum mechanics, it is also possible to evaluate the mass spectra in the case of a spherically symmetric black hole coupled to a massless scalar field. The quantum states of the black hole mass can either increase or decrease with time, depending on the imposed initial conditions [61].

In another application a massless scalar field may also explain the emergence of singularities. For example, a self-similar solution to the Einstein's equations in fourdimensions can be obtained, representing the collapse of a spherically symmetric, minimally coupled, massless, scalar field leading to a Schwarzschild black hole [62].

The process for extracting energy from a rotating blackhole as proposed by R. Penrose many years ago has been considered as a possible explanation for the gamma ray bursts [63]. These are short bursts of gamma rays at the range oh 100's KeV, lasting a few milliseconds to several hundred seconds [64]. However, it was questioned if the extracted energy is sufficient and if it can be sustained in face of other processes going around the black hole, such as the formation of a plasma composed of charged particle pairs. The basic argument is that such plasma may remove sufficient energy to prevent the generation of gamma ray bursts [65]. It has been noted also that the extraction of energy may be followed by the superradiance amplification of amplitude and frequency of the emitted wave, which may result in an additional loss of energy [66].

\section{Concluding Remarks}

Our sampled analysis of the contributions shows that after approximately forty years, research on gravitation and cosmology in Brazil not only stays on the edge of most recent developments, but also has innovated and leaded some lines of research. The majority of contributions are on cosmology, which we interpret as a natural trend resulting from the very large amount of data indicating the existence of a present day accelerated expansion of the universe and the more recent precision measurements of the CMBR by WMAP.

The topology of the universe has been systematically studied in Brazil for more than two decades [67]. We find it particularly relevant that the meeting counted with several contribution on the experimental detection of the topology of the cosmos $[15,18]$, specially because, just two weeks after the meeting, it was announced that the WMAP results point to a specific topology and that it could be experimentally confirmed by the next planned experiments [17].

Still on the experimental facet of the new gravitational physics, we cannot avoid mentioning the collection of reports on the Brazilian Gravitational wave Project. The development of higher frequency oriented spherical detectors of gravitational waves list as one of our outstanding efforts [47]. Our expectative is that in the next meeting we will have some positive data collected from these detectors.

On the theoretical side, we have spotted three relevant contributions: First, the possibility or not of explaining gamma ray bursts by means of Penrose's process of extracting energy from a black hole has the merit of opening a debate on such relevant matter [65]. Secondly, the interesting description of the universe as a dynamical system appeals to a mathematical aspect in which Brazil has given a substantial contribution, namely the theory of dynamical systems [12]. Thus, future joint ventures on "universe dynamics" are expected and should be stimulated. Finally, it has been suggested that the gauge potential associated with the $S U(2)$ triad holonomy group should have a observational character through the gravitational analogue of the Aharonov-Bohm effect [40].

It was an extremely gratifying experience to talk with most of the contributors during the meeting. We only regret that we were unable to comment on many other quality contributions and to be limited to select a just a few topics.

\section{References}

[1] M. Amoroso Costa, Introdução à Teoria da Relatividade. Editora UFRJ, (in Portuguese) Second ed. (1995)

[2] Pedro D. Rache, Relatividade e sua Applicação ao Estudo dos Phenomenos Physicos Precedidos dos Elementos Indispensaveis de Mathematica. Imprensa Oficial de Minas Gerais, Belo Horizonte (in Portuguese) (1932)

[3] M. Schönberg \& S. Chandrasekhar, Astrophysical Jour. 96, 161 (1942) 
[4] A. Lassenby, Cosmic Microwave Background - Recent Results and Their Implications for Cosmology. Braz. J. Phys. This issue (2004)

[5] J. Martin, Braz. J. Phys. 34, 1307 (2004)

[6] R. Caldwell, Braz. J. Phys. 30, 215 (2000)

[7] S. M. Carroll astro-ph/0107571

[8] J. M. F. Maia, Soluções Cosmológicas com Campos Escalares Acoplados, (in Portuguese), (2003); www.sbf1.if.usp.br/ eventos/ enfpc/xxiv/

[9] Ana Helena Campos et al. Preheating in Quintessence Inflation, (in Portuguese) (2003); www.sbf1.if.usp.br/eventos/ enfpc/xxiv/

[10] Daniele, S.M. Alves and G. M. Kremer, Acceleration field of a Universe Modeled as a Mixture of Scalar and Matter Fields, (2003); www.sbf1.if.usp.br/eventos/enfpc/xxiv/ (2003)

[11] R.O. Ramos, The Prevalence of Nonisentropic Expansion in Inflation Models, (2003); www.sbf1.if.usp.br/eventos/ enfpc/xxiv/

[12] H. Pereira and I.D. Soares, Preheating and Turbulence: Echoes of a not so Quiet Universe, (2003); www.sbf1.if.usp.br/eventos/enfpc/xxiv/, see also I. D. Soares, Phys. Rev. D 60, 121301 (1999)

[13] N. Billic et al, The Chaplygin Gas and the Evolution of Dark Matter and Dark Energy in the Universe, astro-ph/0307214, (2003)

[14] M. Makler et al, Observational Constraints on Chaplygin Quartessence: Background Results, astro-ph/0306507, (2003)

[15] M. J. Rebouças and G. I. Gomero, Braz. J. Phys. 34, 1358 (2004)

[16] Titus Lucretius Carus, On the nature of the Universe, circa 50 B.C. Penguin Books, (1951)

[17] J. P. Luminet et al, Nature 425, 593 (2003), astro$\mathrm{ph} / 0310253,(2003)$

[18] B. Mota et al, Constraints on the Detectability of Cosmic Topology from Observational Class. Quant. Grav. 20, 4837 (2003), gr-qc/0308063,

[19] M. Makler et al, Detectability of Cosmic Topology in the Chaplygin Model of dark Matter and Dark Energy Unification, (2003); www.sbf1.if.usp.br/eventos/enfpc/xxiv/

[20] M.E.X. Guimaraes and A.L.N. Oliveira, Phys. Rev. D 67, 123514 (2003)

[21] V. B. Bezerra et al, Gravitational Field Around a Time-like Current Carrying Screwed Cosmic String in Scalar Tensor Theories, hep-th/0306289

[22] V. A. Lorenci and E. S. Moreira Jr. Spinning Strings Cosmic Dislocations and Chronology Protection, gr-qc/0309122

[23] J.J. Halliwell, The interpretations of Quantum Cosmology and the Problem of Time, gr-qc/0208018

[24] J. David Brown and James W. York, Phys. Rev. D40, 3312 (1989)

[25] N. Pinto Neto et al, Green's Functions for Topology Change, gr-qc/0006096

[26] Nelson P. Neto et al, The Accelerated Expansion of the Universe as a Quantum Cosmological Effect. Phys. Lett. A315, 36 (2003), gr-qc/0302112
[27] N. A. Lemos, The Role of Quantum Cosmology in the Chaotic Dynamics of Inflation, (2003); www.sbf1.if.usp.br/eventos/enfpc/xxiv/

[28] H. Minkowski, Space and Time, in The Principles of Relativity, H. A. Lorentz and others, Dover Publications, (1923)

[29] N. Arkani-Hamed et Al, Phys. Lett. B 429, 263 (1998), Phys. Rev. Lett. 84, 586 (2000)

[30] L. Randall \& R. Sundrum, Phys. Rev. Lett. 83, 3370 (1999); Phys. Rev. Lett. 83, 4690 (1999).

[31] R. S. Decca et al, Improved Tests of Extra-dimensional Physics and Thermal Quantum Field Theory From new Casimir Force Measurements, hep-ph/0310157 (2003)

[32] S. Dimopolous and G. Landsberg, Black Hole Production at Future Colliders. SNOWMASS-2001-P321, (2001) (also in Les Houches (2001)). See also, K. Cheung, Collider Phenomenology for Models of Extra Dimensions, hep-ph/0305003, (2003)

[33] M. D. Maia et al, On Friedmann's Equation in Brane-Worlds, Intl. Jour. Mod. Phys. A 17, 29 (2002)

[34] J.C. Fabris, J.A. de Oliveira Marinho, (2003); www.sbf1.if.usp.br/eventos/enfpc/xxiv/

[35] G. Dvali, G. Gabedadze, M. Porrati, Phys. Lett. B485, 208 (2000), hep-th/0005016

[36] T. Damour et al, hep-th/0212155, (2002)

[37] L. C. Bassalo Crispino and G. E. A. Matsas, Radiation Emission from a Uniformly Accelerated Source in n-dimensional Minkowski Spacetime, (2003); www.sbf1.if.usp.br/eventos/enfpc/xxiv/

[38] K. Moriyasu, An Elementary Primer for Gauge Theory, World Scientific, (1983).

[39] Lee Smolin, How Far Are We from the Quantum Theory of Gravity? hep-th/0303185, (2003)

[40] A. M. de Morais Carvalho et al, Holonomy Transformation in a d-Brane, (2003); www.sbf1.if.usp.br/eventos/enfpc/xxiv/

[41] D. Bazeia Filho and J. R. S. Nascimento Tachyons and Braneworlds, (2003); www.sbf1.if.usp.br/eventos/enfpc/xxiv/, hep-th/0306284

[42] R. Durrer and P. Kocian, Testing Brane Worlds with the Binary Pulsar, hep-th/0305181, (2003)

[43] M.D. Maia, Kaluza-Klein Massive Modes in Brane-worlds, (2003); www.sbf1.if.usp.br/eventos/enfpc/xxiv/

[44] A. Y. Miguelote et al, Gravitational Collapse of Self-Similar Perfect Fluid in $2+1$ Gravity, gr-qc/0304035, (2003)

[45] C. M. Zanetti and F. P. Devecchi, Regimes Inflacionário e de Energia Escura em uma Cosmologia Viscosa em 2+1dimensões, (2003); www.sbf1.if.usp.br/eventos/enfpc/xxiv/

[46] M. B. Christmann et al, A Study on Inflation and Dark Energy Regimes in Dilatonic and Jackiw-Teitelboim Cosmologies, (2003); www.sbf1.if.usp.br/eventos/enfpc/xxiv/

[47] O.D. Aguiar et al, The Gravitational Wave detector "MARIO SCHENBERG": Status of the Project Braz. J. Phys. 32, 866 (2002); O. D. Aguiar et al, First Cryogenic Run of the Sphere and Latest Developments, (2003); www.sbf1.if.usp.br/eventos/enfpc/xxiv/ 
[48] Response of the Brazilian gravitational wave Detector to Signals From a Black Hole Ringdown. Cesar A. Costa et al grqc/0309047 (2003)

[49] C. Frajuca et al, Transducers for The Schenberg Detector: Addresing Some Mechanical Challenges, (2003); www.sbf1.if.usp.br/eventos/enfpc/xxiv/

[50] Novello et al, Artificial Black Holes, World Scientific (2002)

[51] V.A. De Lorenci et al, On Optical Black Holes in Moving Dieletrics, Phys. Rev. D 68, 061502, gr-qc/0210104

[52] V.A. De Lorenci and R. Klippert, Electromagnetic Light Rays in Local Dielectrics, (2003); www.sbf1.if.usp.br/eventos/ enfpc/xxiv/

[53] M. L. Bedran, The Effect of Negative Pressure, (2003); www.sbf1.if.usp.br/eventos/enfpc/xxiv/

[54] R. Aldrovandi et al, Gravitation Without the Equivalence Principle, gr-qc/0304106

[55] R. Aldrovandi, J. G. Pereira, and K. H. Vu, Braz. J. Phys. 34, 1374 (2004)

[56] L. O’Raifeartaigh, Phys. Rev. Lett. 14, 475 (1965)

[57] S. Coleman and J. Madula, Phys. Rev. 159, 1251, (1967)

[58] M. D. Maia, Rev. Bras. Fis. 11, 429 (1981)

[59] J.C. Rodryguez et al, Interação da Radiação de Hawking com Fontes Escalares Estáticas nos Espaço-tempos de deSitter e Schwarzschild-deSitter (in portuguese), (2003); www.sbf1.if.usp.br/eventos/enfpc/xxiv/
[60] M. M. Horta Barreira, A Semi-Classical Quantization for Loop Quantum Gravity, (2003); www.sbf1.if.usp.br/eventos/ enfpc/xxiv/

[61] J. Acacio de Barros et al, Causal Interpretation of Spherically Symmetric Evaporating Black Holes, (2003); www.sbf1.if.usp.br/eventos/enfpc/xxiv/

[62] G. Oliveira-Neto and F. I. Takakura, Formation of Schwarzschild Black Hole From the Scalar Field Collapse in Four Dimensions, (2003); www.sbf1.if.usp.br/eventos/enfpc/xxiv/

[63] R. Rufini et al, New perspectives in Physics and Astrophysics from the Theoretical Understanding of Gamma-Ray Bursts, "Proceedings of the $X^{\text {th }}$ Brazilian School of Cosmology and Gravitation", M. Novello, editor, AIP, in press, astro$\mathrm{ph} / 0302557$,

[64] T. Piran, Gamma-ray Bursts: A Primer for Relativists, grqc/0205045

[65] R. Opher, Can Black Holes Supply the Energy Needed for Quasars and Gamma Gay Bursts?, (2003); www.sbf1.if.usp.br/eventos/enfpc/xxiv/

[66] S. Joras and F. D. Sasse, Scalar Wavepacket Superradiance, (2003); www.sbf1.if.usp.br/eventos/enfpc/xxiv/

[67] H. V. Fagundes, On The Visualization of Bolyai - Lobatchevsky's Geometry, IFT P 03/78-São Paulo, (1978). Ibid, The Intuition of Curved Spaces. IFT-P 12/80-São Paulo, (1980) 